\title{
Gut colonisation process of newborns and breast-fed babies at risk of developing coeliac disease
}

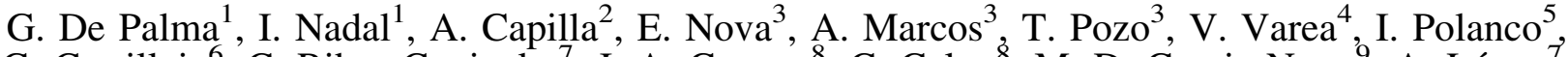 \\ G. Castillejo ${ }^{6}$, C. Ribes-Coninckx ${ }^{7}$, J. A. Garrote ${ }^{8}$, C. Calvo ${ }^{8}$, M. D. Garcia-Novo9 ${ }^{9}$, A. López ${ }^{7}$, \\ M. L. Cilleruelo ${ }^{10}$, F. Palau ${ }^{2}$ and Y. Sanz \\ ${ }^{1}$ Instituto de Agroquímica y Tecnología de Alimentos (CSIC), P.O. Box 73, 46100 Burjassot-Valencia, Spain, ${ }^{2}$ Instituto \\ de Biomedicina de Valencia (CSIC), Valencia, Spain, ${ }^{3}$ Instituto del Frío-ICTAN (CSIC), Madrid, Spain, ${ }^{4}$ Gastroenterología, \\ Nutrición y Hepatología Pediátrica, Hospital Universitario Sant Joan de Deu and Institut Dexeus, Barcelona, Spain, \\ ${ }^{5}$ Servicio de Gastroenterologia y Nutrición Pediátrica, Hospital Universitario La Paz, Madrid, Spain, ${ }^{6}$ Unidad \\ de Gastroenterología Pediátrica, Hospital Universitario Sant Joan de Reus, Tarragona, Spain, ${ }^{7}$ Unidad \\ de Gastroenterologia, Hospital Infantil Universitario La Fe, Valencia, Spain, ${ }^{8}$ Research Unit and Paediatrics Service, \\ Hospital Clínico Universitario, Valladolid, Spain, ${ }^{9}$ Unidad de Gastroenterologia, Hospital Universitario Infantil Niño Jesús, \\ Madrid, Spain and ${ }^{10}$ Servicio de Pediatría, Hospital Severo Ochoa, Leganés, Madrid, Spain
}

Coeliac disease (CD) is an enteropathy triggered by the ingestion of cereal gluten proteins. CD is strongly associated with the human leucocyte antigen (HLA) genes of the major histocompatibility complex genomic region; however, only a small percentage (20-50\%) of subjects bearing the high risk HLA haplotypes develop $\mathrm{CD}^{(1)}$. The microbiota rapidly colonises the gut after birth and strongly influences the health status. In the context of $\mathrm{CD}$, differences in the composition of the gut microbiota have been detected between patients and healthy controls ${ }^{(2)}$, but the role played by the microbiota in the disease risk is unknown. The objective of this preliminary study was to determine whether the gut colonisation pattern of breast-fed babies could be influenced by the genetic CD risk based on their HLA-DQ status. Twenty newborns with at least one first-degree relative with CD were classified in different genetic risk groups by PCR-SPP DQB1 and DQA1 typing. The faecal microbiota was analysed at 7 days, 1 and 4 months of children's age by fluorescent in situ hybridisation and flow cytometry. According to the HLA status, the children were classified into three genetic risk groups. The first group assembled all individuals with DQ2 haplotype in cis or trans conformation with high probability to develop CD (>20\%); the second, those with DQ2 haplotype along with any other haplotype and an intermediate probability to develop CD (1-10\%); and the third group, those with all other genotypes and low probability to develop CD $(<1 \%)$. Total Gram-negative bacteria and Bacteroides-Prevotella group proportions were higher $(P<0.05)$ in the high than in the intermediate and low genetic risk groups. Escherichia coli, Streptococcus-Lactococcus, Eubacterium rectale-Clostridium coccoides group proportions were also significantly higher $(P<0.05)$ in the high than in the low genetic risk group. Similarly, Streptococcus-Lactococcus $(P<0.05)$, E. rectale-C. coccoides $(P<0.05)$ and Bacteroides - Prevotella $(P<0.10)$ groups proportions were higher when the genetic risk to develop CD was high than low at 4 months of age. In summary, the obtained results suggest a relationship between HLA-DQ genes and the gut microbial colonisation process that could lead to a change in the way this disorder and its therapy are investigated.

This work was supported by grants AGL2007-66126-C03-01, 02 and 03/ALI and Consolider Fun- $C$-Food CSD2007-00063 from the Spanish Ministry of Science and Innovation, 200570F0091 from CSIC and CIBERER from the Instituto de Salud Carlos III. The scholarship to G. De Palma from CSIC and to I. Nadal from CSIC (200570F0091) and G.V. are acknowledged. J.A. Garrote is contracted by Fundación Instituto de Estudios de Ciencias de la Salud de Castilla y León (IECSCYL).

1. Mearin ML, Ivarsson A \& Dickey W (2005) Best Pract Res Clin Gastroenterol 19, 441-452.

2. Nadal I, Donant E, Ribes-Koninckx C et al. (2007) J Med Microbiol 56, 1669-1674. 